\title{
EEMD-MUSIC-Based Analysis for Natural Frequencies Identification of Structures Using Artificial and Natural Excitations
}

\author{
David Camarena-Martinez, ${ }^{1}$ Juan P. Amezquita-Sanchez, ${ }^{1}$ Martin Valtierra-Rodriguez, \\ Rene J. Romero-Troncoso, ${ }^{2}$ Roque A. Osornio-Rios, ${ }^{1}$ and Arturo Garcia-Perez ${ }^{2}$ \\ ${ }^{1}$ HSPdigital-CA Mecatronica, Facultad de Ingenieria, Universidad Autonoma de Queretaro, Campus San Juan del Rio, \\ Rio Moctezuma 249, 76807 San Juan del Rio, QRO, Mexico \\ ${ }^{2}$ HSPdigital-CA Telematica, Procesamiento Digital de Señales, DICIS, Universidad de Guanajuato, Carr. Salamanca-Valle km 3.5+1.8, \\ Palo Blanco, 36700 Salamanca, GTO, Mexico \\ Correspondence should be addressed to Arturo Garcia-Perez; agarcia@hspdigital.org
}

Received 12 October 2013; Accepted 22 December 2013; Published 10 February 2014

Academic Editors: S. Berretti and V. Ponomaryov

Copyright (C) 2014 David Camarena-Martinez et al. This is an open access article distributed under the Creative Commons Attribution License, which permits unrestricted use, distribution, and reproduction in any medium, provided the original work is properly cited.

\begin{abstract}
This paper presents a new EEMD-MUSIC- (ensemble empirical mode decomposition-multiple signal classification-) based methodology to identify modal frequencies in structures ranging from free and ambient vibration signals produced by artificial and natural excitations and also considering several factors as nonstationary effects, close modal frequencies, and noisy environments, which are common situations where several techniques reported in literature fail. The EEMD and MUSIC methods are used to decompose the vibration signal into a set of IMFs (intrinsic mode functions) and to identify the natural frequencies of a structure, respectively. The effectiveness of the proposed methodology has been validated and tested with synthetic signals and under real operating conditions. The experiments are focused on extracting the natural frequencies of a truss-type scaled structure and of a bridge used for both highway traffic and pedestrians. Results show the proposed methodology as a suitable solution for natural frequencies identification of structures from free and ambient vibration signals.
\end{abstract}

\section{Introduction}

Nowadays, the vibration-based structural health monitoring (SHM) is a major and fast growing research discipline for several fields such as mechanical engineering, aeronautics, and civil engineering, among others, because it allows the examination of the dynamic characteristics of a specific structure. The basic idea in vibration-based SHM is that physical variations in the structure change its modal parameters and, consequently, its vibration response also experiments disturbances. Therefore, accurate identification and measurement of modal parameters such as natural frequencies are fundamental in vibration-based SHM in order to estimate different structural conditions $[1,2]$. Moreover, a correct identification of modal parameters allows building a proper analytical model, as well as determining the existence and location of structural damage, and in some cases calculating the lifetime of the structure [3]. Several vibration excitation sources have been used to excite civil structures in order to measure their modal properties, which can be classified into two groups. The first one named artificial excitation uses mechanical shakers, drop weights, shoot rockets, control devices, and so on. The second group, named natural excitation, uses ambient vibrations such as wind and earthquakes. The ambient vibration testing has the advantage of being inexpensive and not interrupting the normal operation since no excitation equipment and traffic interruption are needed and also has potentials for implementing real-time condition assessment [4-6]. However, the modal parameter identification of civil engineering structures using the field ambient vibration 




FIgURE 1: Proposed methodology. (a) Synthetic signal, (b) artificial excitation, and (c) natural excitation.

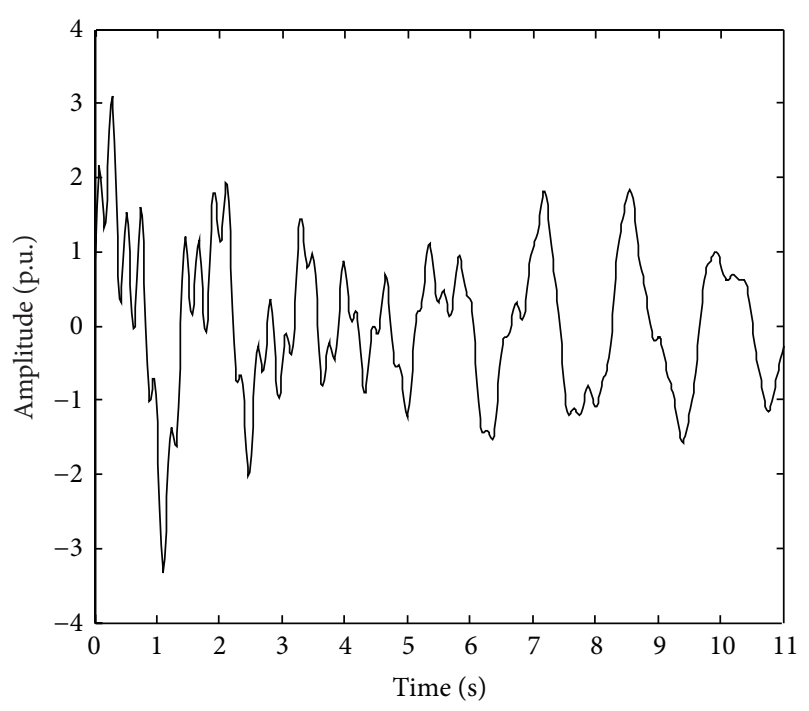

FIGURE 2: Synthetic signal.

measurement is a complicated procedure, since ambient vibration data is nonstationary and it is embedded in highlevel noise. Therefore, it is desirable to have a reliable data analysis or signal processing method capable of analyzing ambient vibrations.

In the past few years, a number of different techniques have been proposed to estimate the modal parameters of a structure such as the peak-picking method (PP) [7], the frequency response function (FRF) [8], the natural excitation technique (NexT) [9], the stochastic subspace identification method (SSI) $[10,11]$, the multivariate AR model [12], the autoregressive-moving average model (ARMA) [13], the eigensystem realization algorithm (ERA) [14], the enhanced frequency domain decomposition (EFDD) [15], and the McKelvey frequency domain subspace algorithm [14]. However, these methods assume the structural response is stationary in order to simplify the signal processing, which is not true in many cases, and therefore the modal parameters cannot be adequately analyzed by these methods [4]. Besides, some methods need a large set of data to identify accurately the modal parameters and, moreover, the results may be affected in noisy conditions $[16,17]$. To overcome these drawbacks, new tools, such as the wavelet transform (WT) $[18,19]$, the Hilbert-Huang transform (HHT) [2022], and the empirical mode decomposition (EMD) with random decrement (RD) [4], have been used to estimate the modal parameters of a structure as well as for damage detection. The WT method provides the time-scale analysis of a transient and nonstationary signal since it decomposes the signal into time-scale representation rather than timefrequency representation. Unfortunately, the WT capabilities are significantly degraded in noisy environments, as the case of real applications; besides, it requires many decomposition levels to be able to measure the modal parameters [19]. The HHT provides a time-frequency representation of nonstationary and transient signals. Its characteristics have been demonstrated to be more precise for decomposing a signal in the time-frequency domain than WT [23]. The HHT is based on two-steps, which are the EMD and the Hilbert transform (HT) methodologies. The EMD method decomposes any data into a set of band-limited quasistationary functions, called intrinsic mode functions (IMFs); later, the Hilbert transform is applied to each IMF, which allows obtaining the amplitude and phase angle of each IMF. Regrettably, the major drawback of EMD method is the so-called mode mixing effect, where the mode mixing indicates that oscillations with the same time scale have been assigned to different IMFs [24]. For this reason, [25] proposed the ensemble EMD (EEMD) method in order to solve the problems of mode mixing caused by an intermittent frequency in the EMD method. Despite this, the EEMD-HT, EMD-HT, and EMD$\mathrm{RD}$ methods have some difficulties to detect or extract close space modes, which are present in many civil structures due to the symmetrical geometry and similar physical properties in different directions. In addition, these methods are also very susceptible to noise. Therefore, it would be desirable to have a technique that helps the EEMD to extract the natural frequencies of a structure with great efficiency and accuracy, especially those modes that are very close. Regarding close frequency components, a high-resolution spectral estimation through the multiple signal classification (MUSIC) algorithm has been successfully presented for very close frequency 

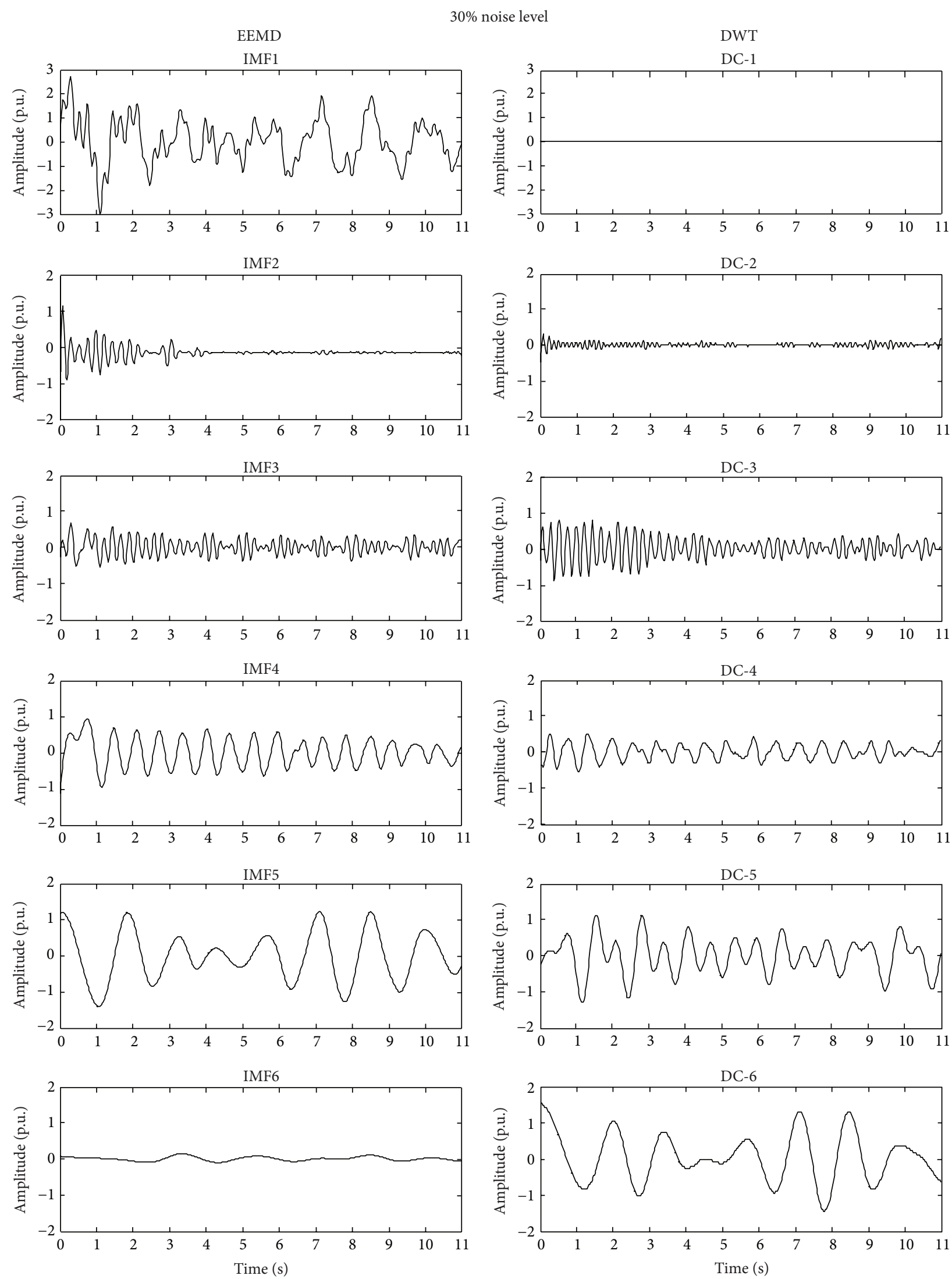

(a)

(b)

FIGURE 3: IMFs and DCs obtained of the synthetic signal through EEMD and DWT, respectively. 


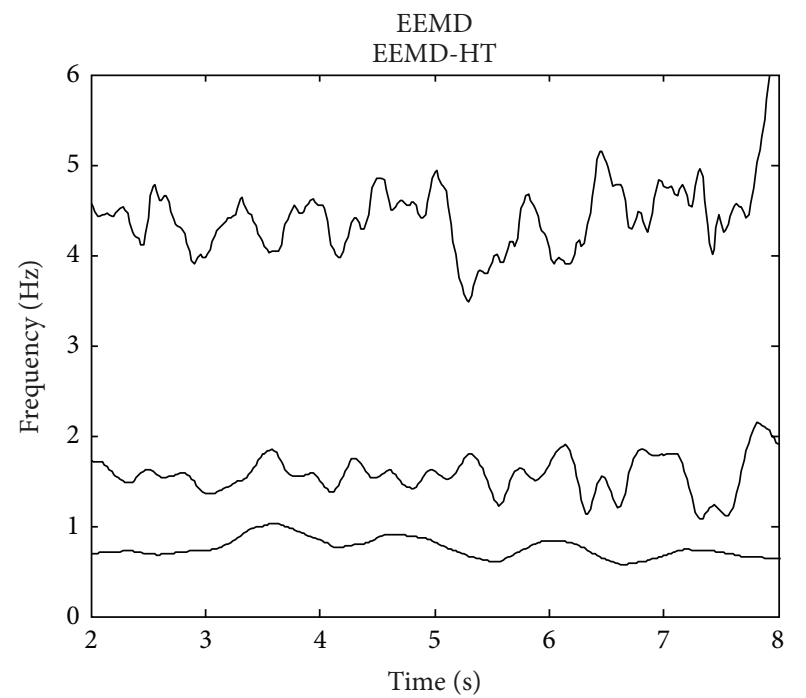

(a)
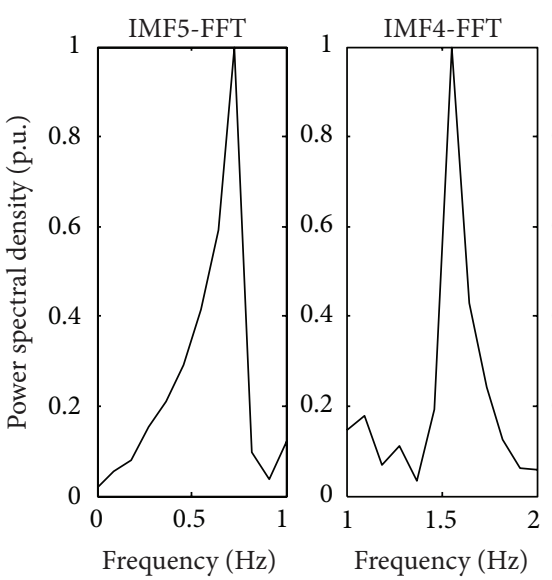

(c)
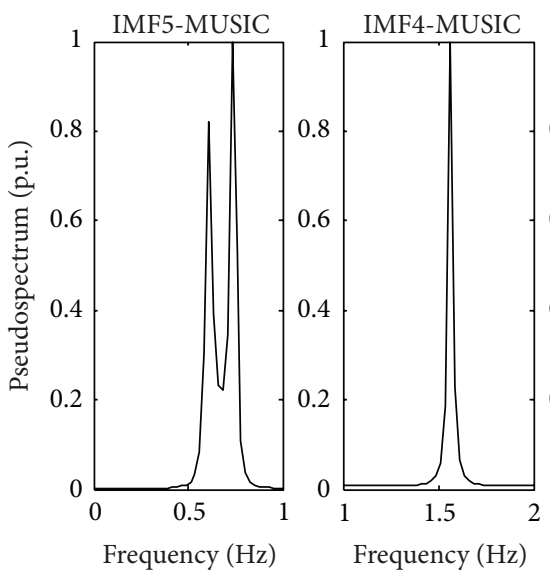

(e)

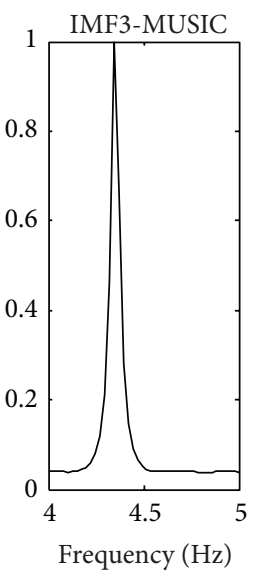

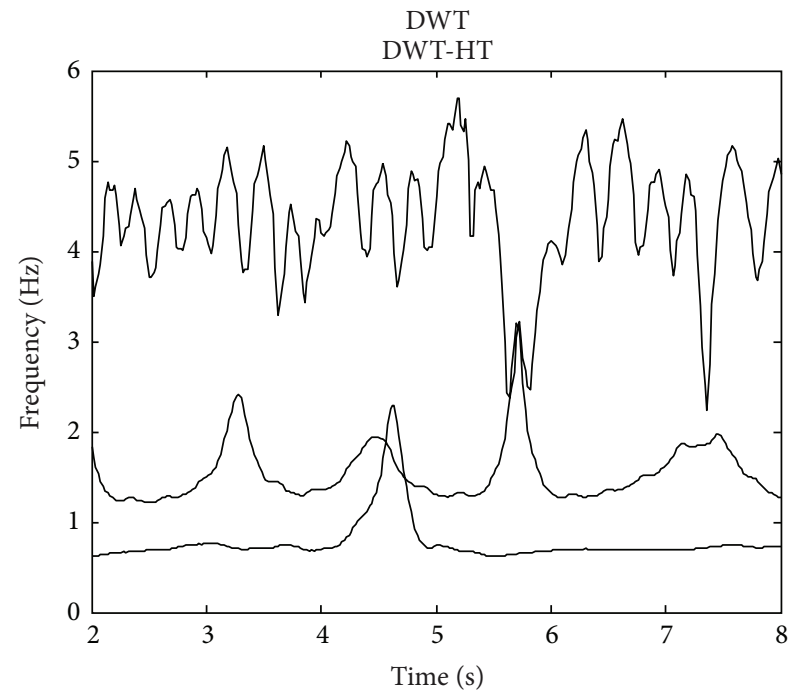

(b)
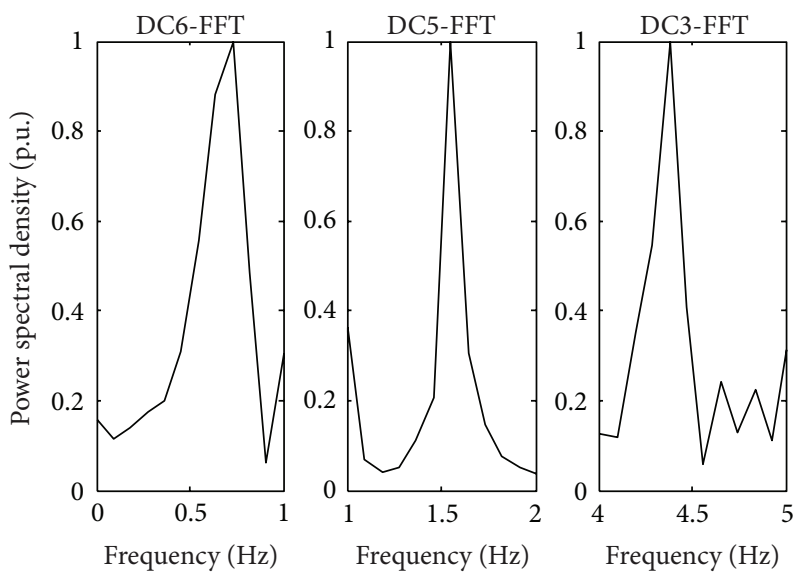

(d)
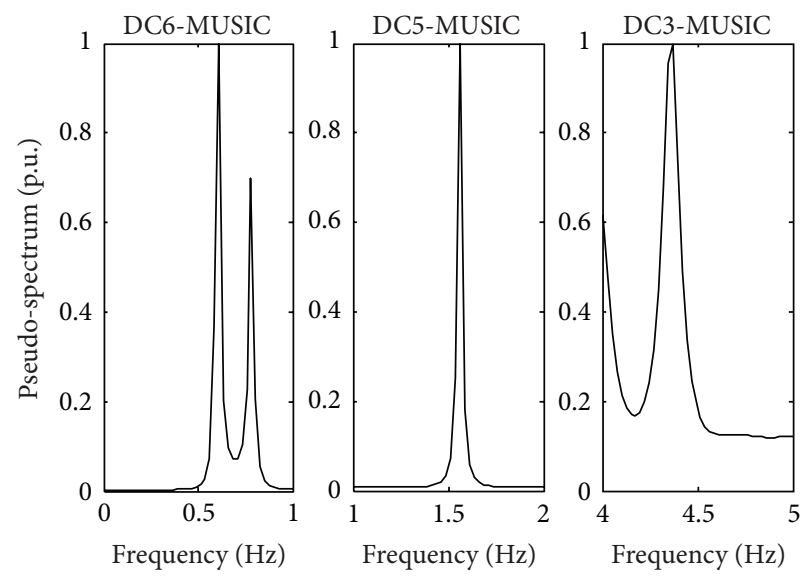

(f)

FIgURe 4: Results obtained by (a) EEMD-HT method, (b) DWT-HT, (c) EEMD-FFT, (d) DWT-FFT, (e) EEMD-MUSIC, and (f) DWTMUSIC. 


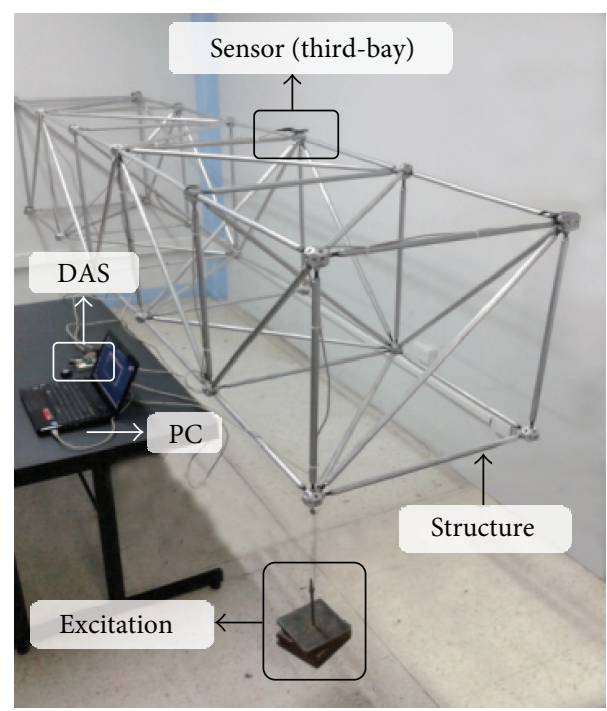

(a)

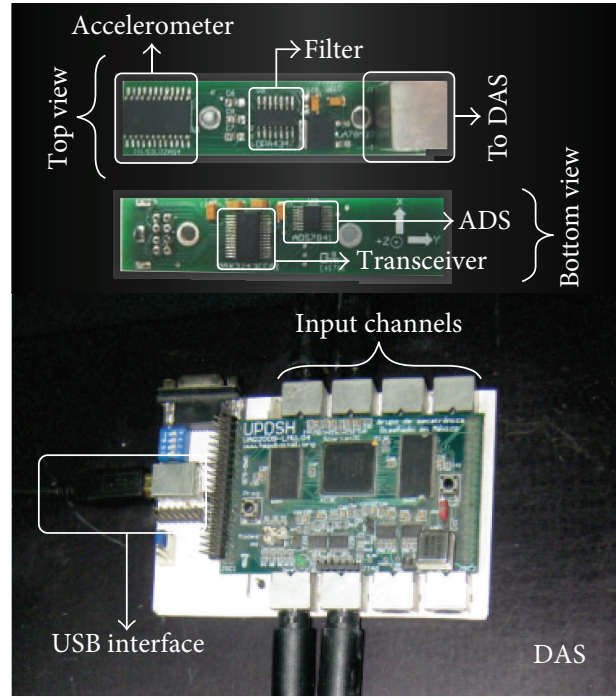

(b)

FIGURE 5: Experimental setup: (a) overall system and (b) triaxial accelerometer and data acquisition system.

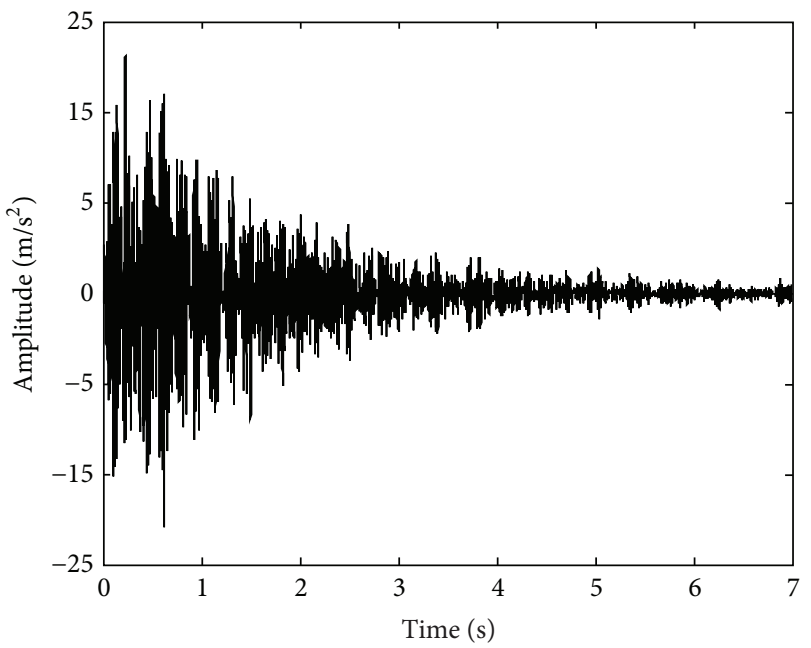

FIGURE 6: Measured vertical acceleration.

components, even for data with low signal-to-noise ratio [26, 27]; therefore, the MUSIC algorithm is a promising technique to detect the close space modes of the structures.

The contribution of this paper is to present a new EEMDMUSIC-based methodology to identify modal frequencies in structures ranging from free and ambient vibration signals produced by artificial and natural excitations. The EEMD is used to decompose the vibration signal into a set of IMFs and then MUSIC identifies the natural frequencies of the structure. The usefulness and effectiveness of the proposed methodology are assessed through three experimental cases. In the first experiment, a numerical simulation is carried out to validate the accuracy and noise immunity of the proposed methodology for extracting the natural frequencies, especially when the modal frequencies are close. The second experiment is focused on extracting the modes of a trusstype scaled structure by using an artificial excitation, where the results are compared with the obtained values through finite element analysis (FEA). Finally, the third experiment concentrates on identifying the natural frequencies of a bridge with natural excitation by using a set of field ambient vibration measurements. Analytical and experimental results show an excellent identification of the natural modes even when the signal is embedded in high-level noise, and, more importantly, the results demonstrate that the methodology provides a suitable alternative approach to natural frequencies identification from free and ambient data by using artificial and natural excitations.

\section{Mathematical Background}

This section presents the mathematical background of the proposed techniques for vibration analysis of structures.

2.1. Empirical Mode Decomposition. The EMD method is a data-processing technique for analyzing nonstationary and nonlinear signals. The EMD method decomposes the vibration signal into a set of band-limited quasistationary functions called IMFs. Each IMF has to satisfy the following two conditions: (i) the number of zero crossings and the number of extrema must either equal or differ by at most one and (ii) the mean value of the envelope defined by the local maxima and the envelope defined by the local minima must be zero [28].

The process for obtaining each IMF is called the sifting process, which is described as follows.

(1) Identify the maxima and minima of the signal $x(t)$.

(2) Generate an upper and lower envelop by using cubic spline interpolation. The average of the two envelops 

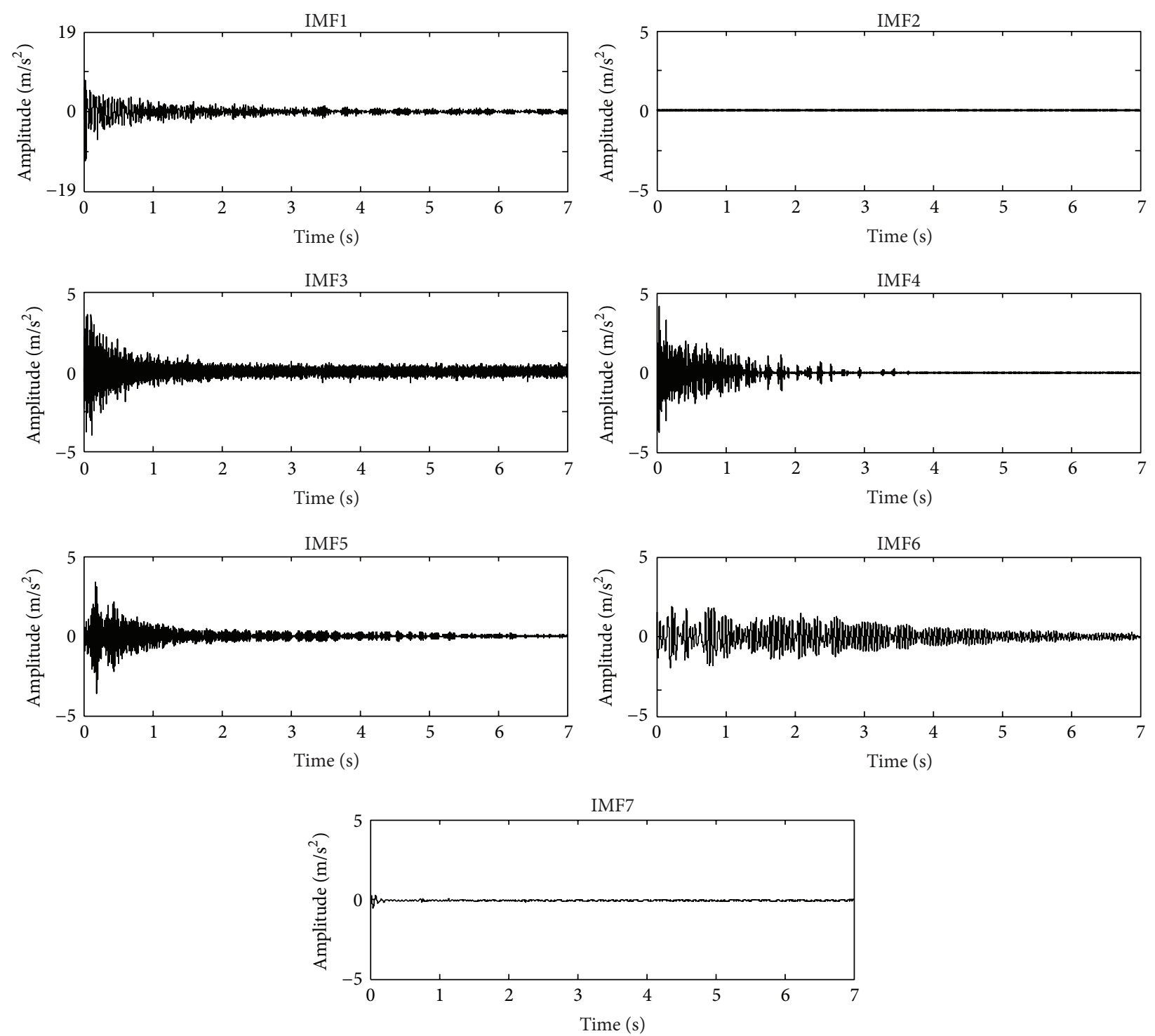

FIGURE 7: IMF obtained through EEMD for free vibration acceleration.

is $m_{1}(t)$. Subtract $m_{1}(t)$ from the original signal $x(t)$ to obtain

$$
h_{1}(t)=x(t)-m_{1}(t) .
$$

Determine if (1) satisfies the conditions (i) and (ii). If not, repeat the first two steps until $h_{k}(t)$ satisfies the conditions (i) and (ii); then, $h_{k}(t)$ is the first IMF defined as

$$
c_{1}(t)=h_{k}(t)=\text { IMF1 }
$$

(3) After IMF1 is obtained, subtract $c_{1}$ from the original signal $x(t)$ and calculate the residue signal as follows:

$$
r_{1}(t)=x(t)-c_{1}(t) .
$$

(4) Treat $r_{1}$ as the original signal and repeat the procedure from step (1) to step (3) to obtain the other IMF $\left(c_{2}, c_{3}, \ldots, c_{n}\right)$. The process is stopped when the final residual signal $r_{n}(t)$ is a monotonic function.

(5) At the end of the procedure, the signal $x(t)$ is decomposed into $n$ intrinsic modes $c_{i}(t)$ and a residue $r_{n}(t)$. Now, the original signal can be represented by

$$
x(t)=\sum_{i=1}^{n} c_{i}(t)+r_{n}(t) .
$$

2.2. Ensemble Empirical Mode Decomposition. The EEMD is an adaptive and noise-assisted method proposed by [25, 29] in order to improve the shortcomings of the EMD algorithm and provide a feasible solution of mode mixing caused by an intermittent frequency. The EEMD method defines the IMF set for an ensemble of trials, each one obtained by applying the EMD to the signal of interest with added independent 

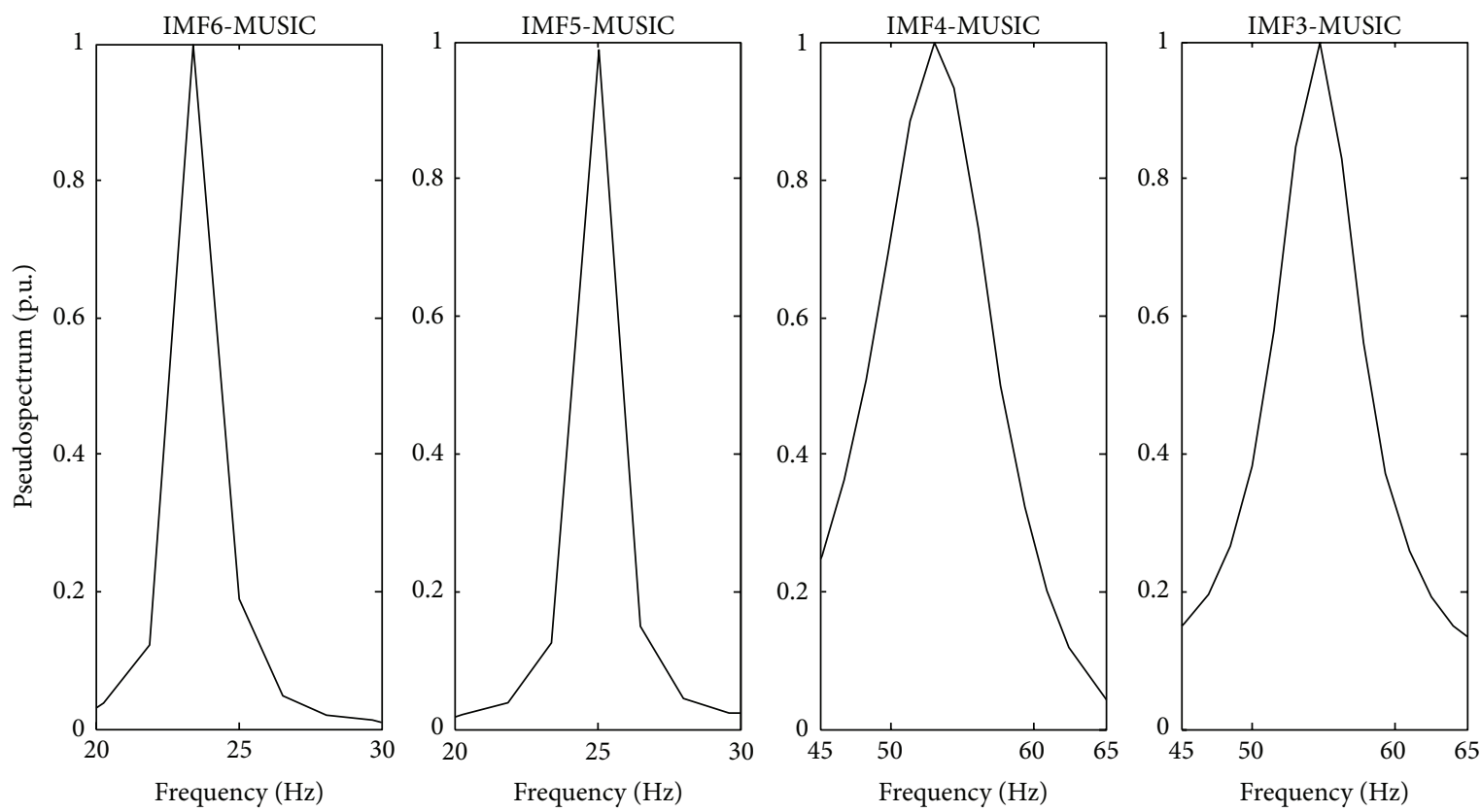

FIGURE 8: Pseudospectra of different analyzed IMF.
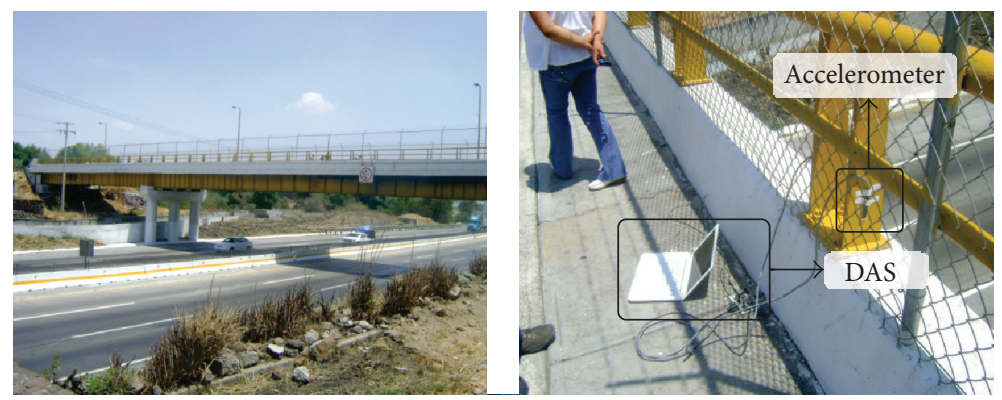

FIgUre 9: Analyzed bridge.

identically distributed white noise of the same standard deviation.

According to [25], EEMD contains the following steps.

(1) Add a white noise series to the targeted data.

(2) Decompose the data with added white noise by EMD algorithm, described in Section 2.1.

(3) Repeat steps (1) and (2) until the trial number is met, but with different white noise series each time.

(4) Estimate the (ensemble) means of corresponding IMF of the decompositions as the desired output.

2.3. MUSIC Algorithm. MUSIC algorithm is known as a high-resolution method which can detect frequencies with low signal-to-noise ratio. MUSIC assumes that the discrete time signal $x[n]$ can be represented as a sum of $m$ complex sinusoid in noise $e[n][30]$. Consider

$$
x[n]=\sum_{i=1}^{m} \overline{B_{i}} e^{j 2 \pi f_{i} n}+e[n], \quad n=0,1,2, \ldots, N-1
$$

with

$$
\overline{B_{i}}=\left|B_{i}\right| e^{\phi_{i}},
$$

where $N, B_{i}$, and $f_{i}$ are the number of sample data, the complex amplitude, and its frequency, respectively, and $e[n]$ is a sequence of white noise with zero mean and a variance $\sigma^{2}$. This method uses the eigenvector decomposition of $x[n]$ to obtain two orthogonal subspaces. The autocorrelation matrix $R$ of the noisy signal $x[n]$ is the sum of the autocorrelation matrices of the pure signal $R_{s}$ and the noisy $R_{n}$ as follows:

$$
R=R_{s}+R_{n}=\sum_{i=1}^{P}\left|B_{i}\right|^{2} e\left(f_{i}\right) e^{H}\left(f_{i}\right)+\sigma_{n}^{2} I,
$$

where $P$ is the number of frequencies, the exponent $H$ denotes Hermitian transpose, $I$ is the identity matrix, and $e^{H}\left(f_{i}\right)$ is the signal vector given by

$$
e^{H}\left(f_{i}\right)=\left\lfloor\begin{array}{llll}
1 & e^{-j 2 \pi f_{i}} & \cdots & e^{-j 2 \pi f_{i}(N-1)}
\end{array}\right\rfloor .
$$




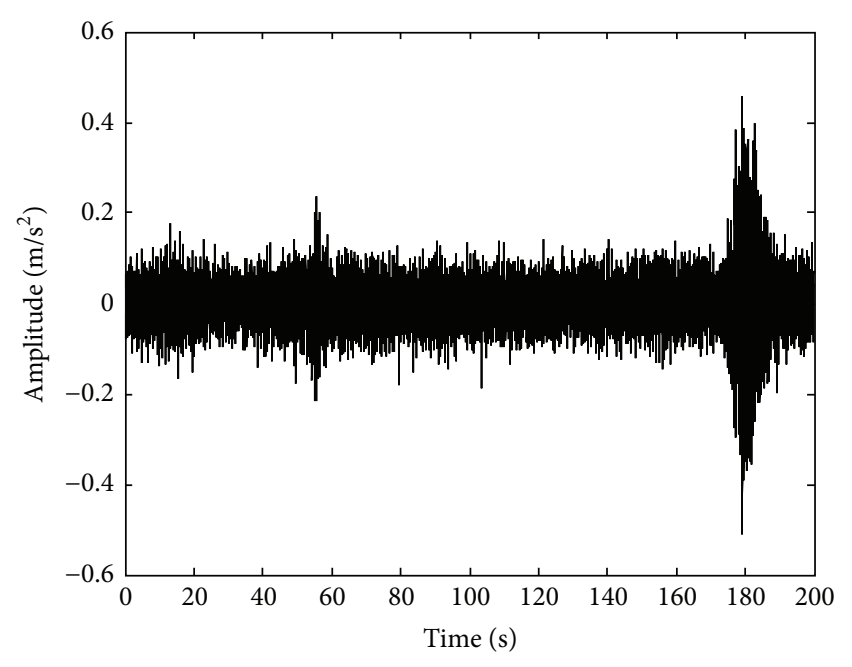

FIGURE 10: Measured acceleration response.

From the orthogonality condition of both subspaces, the MUSIC pseudospectrum $Q$ of the current space vector is given by

$$
Q^{\text {MUSIC }}(f)=\frac{1}{\left|e(f)^{H} V_{m+1}\right|^{2}}
$$

where $V_{m+1}$ is the noise eigenvector. This expression exhibits the peaks that are exactly at frequency of principal sinusoidal component, where $e(f)^{H} V_{m+1}=0$.

\section{Proposed Methodology}

Figure 1 shows the flowchart of the proposed EEMD-MUSICbased methodology for identifying modal frequencies from free and ambient vibration signals produced by an artificial or a natural excitation. The first step is to acquire the vibration signal of the structure. Second, the sampled vibration signal is passed through a band-pass filter, in order to extract the bandwidth of interest related to the modal frequencies as well as to help solve the mode mixing. Third, the filtered vibration signal is decomposed into its IMF utilizing the EEMD. Finally, the MUSIC algorithm is applied to each IMF to compute the modal frequencies of the structure.

The usefulness of the proposed methodology is tested in three experiments. The first experiment focuses on the use of a synthetic signal to validate the accuracy and noise immunity of the proposed methodology (Figure 1(a)). In the second experiment, the proposed methodology is tested on a truss-type scaled structure located in a laboratory, where the excitation conditions are controlled (Figure 1(b)). Finally, the third experiment is carried out to show the effectiveness of the proposed methodology under real operating conditions. In this experiment, a real structure (a bridge) is analyzed, in which the natural excitation is used (Figure 1(c)).

\section{Experimentation and Results}

In order to show the effectiveness of the proposed methodology, three experiments are performed. The first experiment consists of a numerical simulation implemented to validate the accuracy and noise immunity of the proposed methodology for extracting the natural frequencies, especially when the natural frequencies are close. Further, the proposed methodology and different approaches are compared in order to validate their efficiency by estimating the natural frequencies. The second experiment concentrates on an artificial excitation of the free decay under forced vibration that is applied on a truss-type scaled structure for extracting the natural frequencies. The experimental results are compared with those obtained by FEA. Finally, the third experiment concentrates on identifying the natural frequencies of a bridge with natural excitation by using a set of field ambient vibration measurements. This experimentation takes into account that ambient vibration data is nonstationary and it is embedded in high-level noise.

4.1. Synthetic Signal. In order to validate the proposed methodology performance, a comparison between different approaches, EEMD-FFT and -HT, and the discrete wavelet transform (DWT)-FFT, -HT, and -MUSIC methods is presented. For this, a synthetic signal with a priori known values is used, which appears in Figure 2 and corresponds to a damped free vibration response of 4-degree of freedom system as follows:

$$
i(t)=\sum_{j=1}^{4} A_{j} e^{-2 \pi \zeta_{j} f_{j} t} \sin \left(2 \pi f d_{j} t+\theta_{j}\right)+\omega(t),
$$

where $A_{j}, \theta_{j}, f_{j}, f d=f_{j} \sqrt{1-\zeta_{j}^{2}}$, and $\zeta_{j}$ are the amplitude, phase, undamped natural frequency, damped natural frequency, and damping ratio of the $j$ th mode, respectively, whereas $\omega(t)$ is a sequence of white noise. The used numerical values are $f_{1}=0.61 \mathrm{~Hz}, f_{2}=0.73 \mathrm{~Hz}, f_{3}=1.56 \mathrm{~Hz}$, $f_{4}=4.35 \mathrm{~Hz}, \zeta_{j}=0.01, A_{j}=1.0$, and $\theta_{j}=0$, for $j=1$, 2,3 , and 4 , with a sampling frequency and sampling time of $50 \mathrm{~Hz}$ and $11 \mathrm{~s}$, respectively. Besides, a 30\% level noise for an $\mathrm{SNR}=7.4 \mathrm{~dB}$ is used to test the efficiency and immunity of the techniques to noisy environments.

Firstly, the synthetic signal is decomposed, as shown in Figure 3, by the EEMD and DWT which is also a technique to decompose a signal into frequency bands named decompositions (DCs) [3]. Then, some IMFs and DCs are chosen for being analyzed. Regarding the IMFs (Figure 3(a)), the first two IMF levels are discarded since they contain the noise and high-frequency components; therefore, only the IMF3, IMF4, and IMF5 are considered to identify the natural modes. On the other hand and according to the DCs (Figure 3(b)), the first two decompositions, $\mathrm{DC}_{1}$ and $\mathrm{DC}_{2}$, are discarded since they contain the high-frequency bands; consequently, the $\mathrm{DC}_{3}(3.125-6.25 \mathrm{~Hz}), \mathrm{DC}_{5}(0.781-$ $1.5625 \mathrm{~Hz})$, and $\mathrm{DC}_{6}(0.390-0.781 \mathrm{~Hz})$ are analyzed to identify 

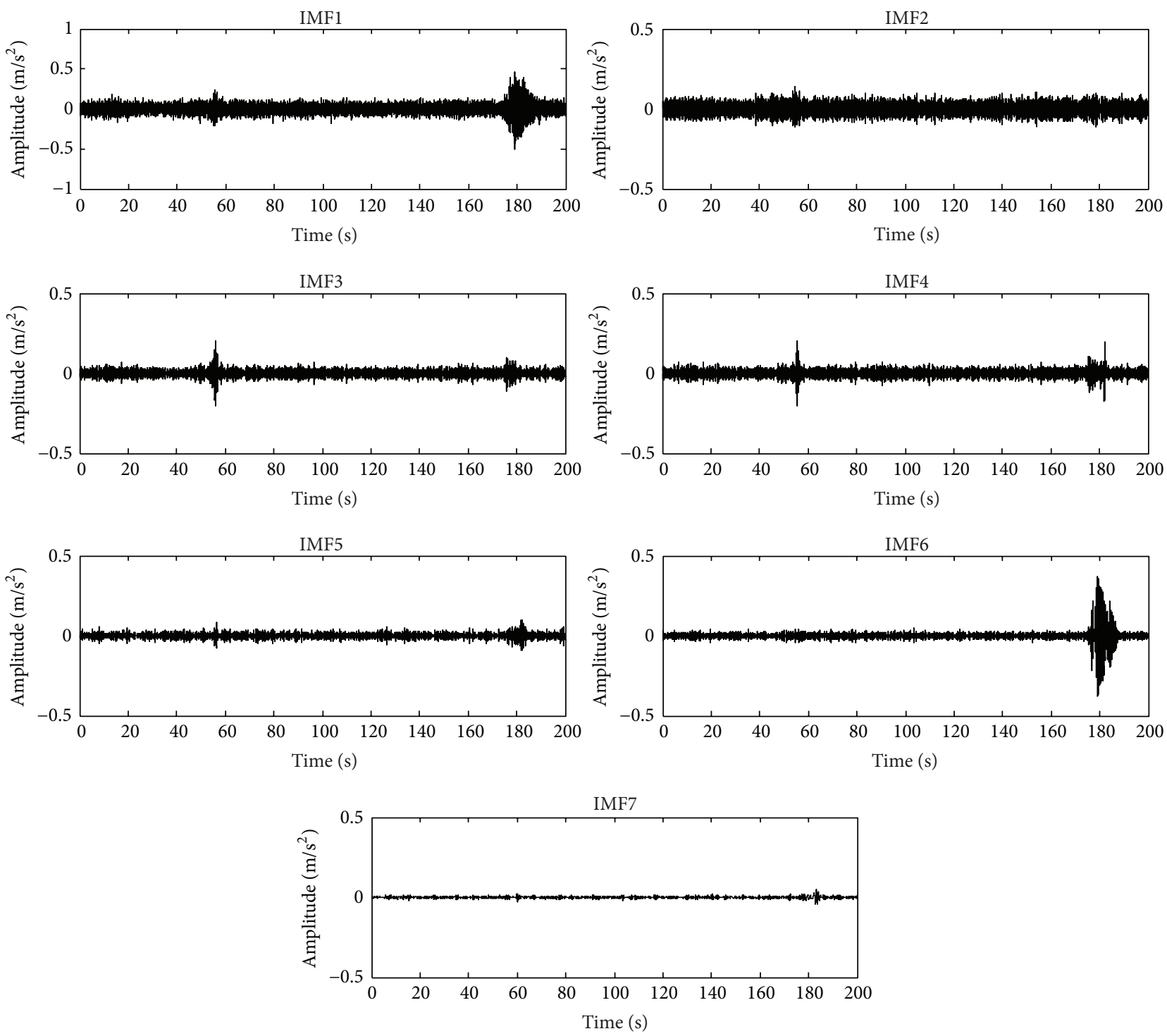

FIGURE 11: IMFs obtained through EEMD for ambient vibration acceleration.

TABLE 1: Results of identified natural frequencies with different methodologies.

\begin{tabular}{|c|c|c|c|c|c|c|}
\hline \multirow{2}{*}{ Reference values } & \multicolumn{3}{|c|}{ EEMD } & \multicolumn{3}{|c|}{ DWT } \\
\hline & $\mathrm{HT}$ & FFT & MUSIC & HT & FFT & MUSIC \\
\hline$f_{1}=0.61$ & \multirow[t]{2}{*}{$0.72^{*}$} & \multirow[t]{2}{*}{$0.68^{*}$} & 0.61 & \multirow[t]{2}{*}{$0.70^{*}$} & \multirow[t]{2}{*}{$0.72^{*}$} & 0.61 \\
\hline$f_{2}=0.73$ & & & 0.73 & & & 0.78 \\
\hline$f_{3}=1.56$ & 1.54 & 1.52 & 1.56 & 1.31 & 1.55 & 1.56 \\
\hline$f_{4}=4.35$ & 4.38 & 4.30 & 4.34 & 4.41 & 4.38 & 4.37 \\
\hline
\end{tabular}

* Mixed modes.

the natural frequencies since they contain the frequencies of interest.

Later, the selected IMFs and DCs are processed by the HT, the FFT, and the proposed MUSIC method as shown in Figure 4. From these figures, it is possible to observe that the EEMD-HT method (Figure 4(a)) and the DWT-HT method
(Figure 4(b)) only detect three modes due to the fact that (i) the EEMD and DWT cannot separate close frequencies and (ii) that the HT can only identify one frequency by each IMF and DC, respectively. Similarly, the EEMD-FFT (Figure 4(c)) and the DWT-FFT (Figure 4(d)) cannot identify close modes due to overlapping frequency components and to 

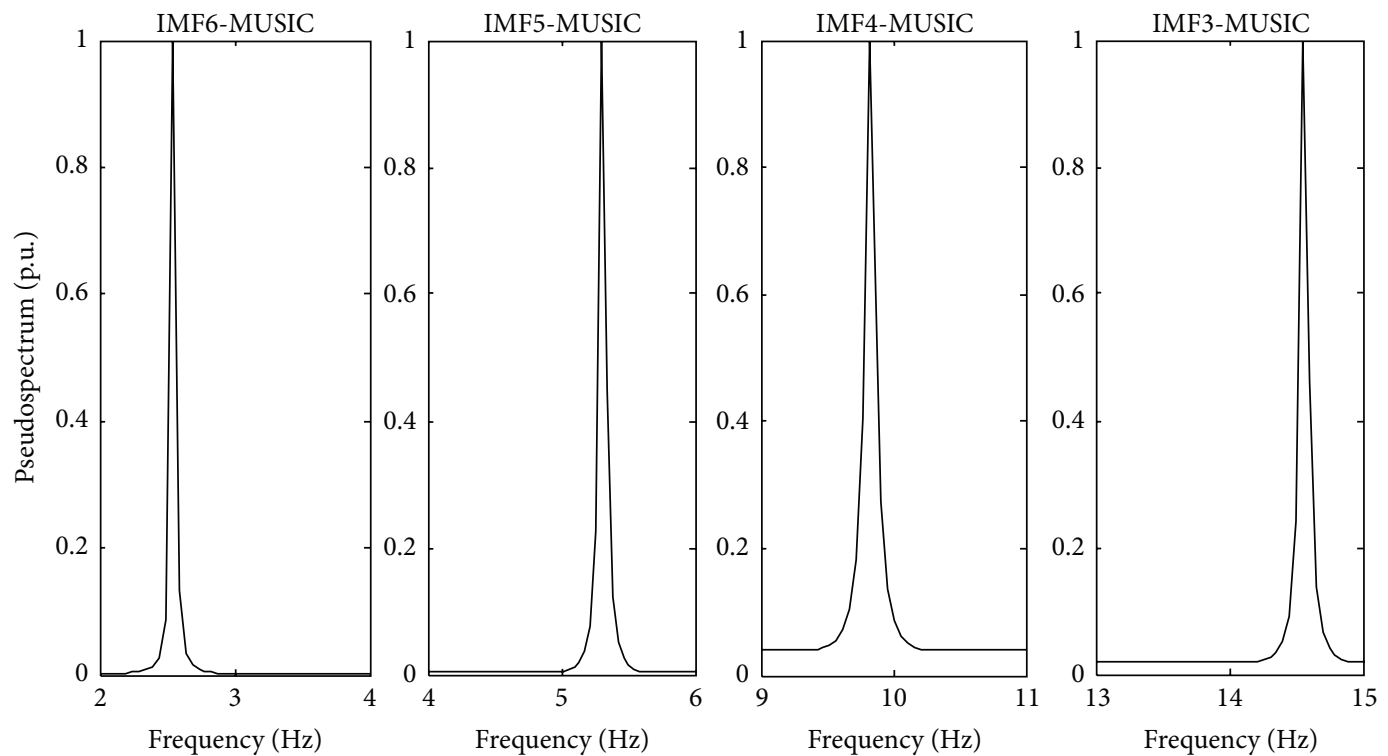

Figure 12: Pseudospectra of different analyzed IMFs.

TABLE 2: Comparison of natural frequencies.

\begin{tabular}{lccc}
\hline Modes & Finite element analysis & $\begin{array}{c}\text { Proposed methodology } \\
\text { (EEMD-MUSIC) }\end{array}$ & DWT-MUSIC \\
\hline & & Natural frequency (Hz) & \\
2 & 24.48 & 23.44 & 26.86 \\
3 & 25.50 & 25.00 & 50.00 \\
4 & 52.57 & 51.56 & 53.13 \\
\hline
\end{tabular}

the FFT resolution. On the other hand, the EEMD-MUSIC (Figure 4(e)) and the DWT-MUSIC (Figure 4(f)) identi-fy clearly the four natural modes; however, the results for the natural frequencies obtained by DWT-MUSIC are degraded in noisy environments unlike the EEMD-MUSIC method. All the aforementioned results are presented in a normalized way for a better presentation and understanding.

Finally, Table 1 shows the numerical comparison between the reference values of the synthetic signal and the obtained values through the EEMD-HT, the EEMD-FFT, the DWTHT, the DWT-FFT, the DWT-MUSIC, and the proposed EEMD-MUSIC methods. As a result of the capabilities of the EEMD-HT, the DWT-HT, the EEMD-FFT, and the DWTFFT methods, the closest natural modes of the synthetic signal, $f_{1}$ and $f_{2}$, are mixed and, therefore, only one value of them is obtained. On the other hand, the proposed EEMDMUSIC method proved to be able to extract the four natural modes with higher accuracy than DWT-MUSIC since the results are affected by the noise level.

The overall proposed methodology for this and the two next study cases is implemented in the MATLAB Digital Signal Processing Toolbox. The MUSIC algorithm has an order of 4 and overlaps of $25 \%$. Regarding the EEMD, the number of ensemble members is set to 100 and the standard deviation of white-noise series is set to 0.1 . On the other hand, the Daubechies ( $\mathrm{db} 10$ ) window is used as the mother wavelet in the DWT.

4.2. Artificial Excitation. In the second experiment, a tridimensional truss-type scaled structure, shown in Figure 5(a), is used for extracting the natural frequencies by means of an artificial excitation. The modal testing is performed using a hanging mass of $14 \mathrm{~kg}$ as excitation source. The mass is tied in the first-bay in order to have the maximum displacement of the structure; then, the wire that subjects the mass is cut to induce a free vibration in the structure. The vibration signal is acquired using a MEMS-based triaxial accelerometer model LIS3L02AS4 from STMicroelectronics placed on top of the third bay as shown in Figure 5(a). The accelerometer has a user-selectable full scale of $\pm 19.6 \mathrm{~m} / \mathrm{s}^{2} / \pm$ $58.8 \mathrm{~m} / \mathrm{s}^{2}$ with a $50 \times 10^{-4} \mathrm{~m} / \mathrm{s}^{2}$ resolution over $100 \mathrm{~Hz}$. The accelerometer information is digitalized using a 12-bit 4channel ADS7841 analog-to-digital converter (ADC) shown in Figure 5(b) from Texas Instruments, with a maximum sampling rate of $200 \mathrm{kHz}$ in each channel. The obtained signals from the accelerometer are stored in a proprietary data acquisition system (DAS) and sent to a personal computer (PC) by universal serial bus (USB) protocol as shown in 
Figure 5(b). The DAS uses a sampling frequency of $3.2 \mathrm{kHz}$ for obtaining 22,400 samples during a time window of $7 \mathrm{~s}$. The vertical free vibration signal, shown in Figure 6, is used to identify the vertical natural frequencies of the structure.

Figure 7 shows the first seven IMFs obtained from the free vibration response signals. The IMF1 and IMF2 contain noise and high-frequency components out of the frequency range of interest. On the other hand, from IMF3 to IMF6 are used for analysis with the MUSIC method because they contain the natural frequencies for the truss-type structure. The rest of the IMFs are discarded since they do not contain relevant information. Figure 8 displays the normalized pseudospectrum obtained from selected IMF, where each one of the natural frequencies is observed. The identification results by the proposed methodology are compared to those from FEA as well as from the experimental result identified with the DWT-MUSIC method. The obtained results are presented in Table 2. It is worth noticing that only the DWT-MUSIC is used as an experimental comparative technique since it has the second best performance after EEMD-MUSIC, as shown in the previous study case. The proposed methodology identifies the natural frequencies reasonably closed when it is compared with the analytical estimation from the FEA. Evidently the small differences may be due to a number of real conditions that are not considered or completely represented in the FEA. On the contrary, the DWT-MUSIC method has a higher error in the identification of the natural frequencies due to the real noisy environment.

4.3. Natural Excitation. The bridge considered in this study is located in "San Juan del Rio, Queretaro, Mexico." The bridge is used for both highway traffic and pedestrians as shown in Figure 9. The ambient vibration caused by traffic is online monitored through a triaxial accelerometer mounted in the middle of the bridge. The obtained signals from the accelerometer are stored in the DAS and sent to PC by USB protocol. The DAS uses a sampling frequency of $160 \mathrm{~Hz}$ for obtaining 32,000 samples during a time window of $200 \mathrm{~s}$, where the vertical vibration response is plotted in Figure 10, and the filtered signal is analyzed through the EEMD method, and its results are shown in Figure 11. The IMF3 to IMF6 are selected to obtain the natural frequencies since it contains the interested frequency range of the bridge. Figure 12 displays the normalized pseudospectra obtained from selected IMF, where each one of the natural frequencies is observed $(2.539 \mathrm{~Hz}, 5.313 \mathrm{~Hz}, 9.814 \mathrm{~Hz}$, and $14.55 \mathrm{~Hz}$ ).

Table 3 shows a comparison between the values obtained from the proposed methodology and the results obtained by the DWT-MUSIC method. This method can detect the four natural frequencies; however, their accuracy is expected to be lower due to the noise, since ambient vibration data is embedded in high-level noise.

\section{Conclusions}

In this paper, a new methodology based on the fusion of the EEMD and the MUSIC methods for identifying the natural frequencies of civil structures from free and ambient
TABLE 3: Identified natural frequencies.

\begin{tabular}{lcc}
\hline Modes & Proposed methodology & DWT-MUSIC \\
\hline & Natural frequencies $(\mathrm{Hz})$ & \\
1 & 2.539 & 2.50 \\
2 & 5.313 & 5.15 \\
3 & 9.814 & 10.00 \\
4 & 14.55 & 14.69 \\
\hline
\end{tabular}

vibration response signals produced by artificial and natural excitations is presented. The effectiveness of the proposal is demonstrated through three experiments. (i) The numerical simulation shows that the proposal can identify closely spaced frequencies even in noisy environments unlike the EEMD-HT, DWT-HT, EEMD-FFT, and DWT-FFT methods; although the DWT-MUSIC method is able to detect close modes, it is sensitive to noisy environments, as the case of free and ambient vibration signals, which does not make it suitable for identifying accurately the natural modes, which is essential in the design of civil structures [31]. Besides, the decomposition level of the DWT has to be known or at least supposed a priori in order to have an adequate result, which is very difficult in real applications since all the signals may have different modes; consequently, the frequency band that contains the natural mode is also different. On the other hand, the EEMD does not have this drawback since it is an intuitive, unsupervised, and self-adaptive method that gives an automatic result. (ii) The very similar obtained natural frequencies through the proposal and FEM of a real truss-type structure make the proposal a suitable tool for the analysis of free vibration signals produced by artificial excitations. (iii) EEMD-MUSIC is a powerful approach for identifying natural frequencies in real and non-controlled civil structures (i.e. a bridge) from ambient vibration data, produced by natural excitations. which is a complicated task since they are nonstationary and embedded in high-level noise.

The immunity to noisy environments and the capability to identify closely spaced frequencies with high accuracy make the proposal a suitable and powerful tool for different research disciplines such as SHM, structural damage detection, and design structure, helping in the development of appropriate and accurate analytical models for improving the performance, resistance, and life service of the structures, among others.

\section{Conflict of Interests}

The authors declare that there is no conflict of interests regarding the publication of this paper.

\section{Acknowledgments}

This work was partially supported by CONACyT scholarship 229795 and SEP PIFI-2013 Universidad de Guanajuato projects. 


\section{References}

[1] S. Doebling, C. Farrar, M. Prime, and D. Shevitz, "Damage identification and health monitoring of structural and mechanical systems from changes in their vibration characteristics: a literature review," Los Alamos National Laboratory Report LA 13070-MS, 1996.

[2] X. Bai and X. Zhang, "A novel evaluation method for building construction project based on integrated information entropy with reliability theory," The Scientific World Journal, vol. 2013, Article ID 573014, 8 pages, 2013.

[3] J. P. Amezquita-Sanchez, R. Osornio-Rios, R. Romero-Troncoso, and A. Dominguez-Gonzalez, "Hardware-software system for simulating and analyzing earthquakes applied to civil structures," Natural Hazards and Earth System Science, vol. 12, no. 1, pp. 61-73, 2012.

[4] X. H. He, X. Hua, Z. Chen, and F. Huang, "EMD-based random decrement technique for modal parameter identification of an existing railway bridge," Engineering Structures, vol. 33, no. 4, pp. 1348-1356, 2011.

[5] W. Shi, J. Shan, and X. Lu, "Modal identification of Shanghai World Financial Center both from free and ambient vibration response," Engineering Structures, vol. 36, pp. 14-26, 2012.

[6] M. Pieraccini, "Monitoring of civil infrastructures by interferometric radar: a review," The Scientific World Journal, vol. 2013, Article ID 786961, 8 pages, 2013.

[7] W. Hu, Á. Cunha, E. Caetano, F. Magalhães, and C. Moutinho, "LabVIEW toolkits for output-only modal identification and long-term dynamic structural monitoring," Structure and Infrastructure Engineering, vol. 6, no. 5, pp. 557-574, 2010.

[8] Z. Ibrahim and P. Reynolds, "Modal testing of a cantilever grandstand," in Proceedings of the International Conference on Construction and Building Technology (ICCBT '08), pp. 271-284, Kuala Lumpur, Malaysia, June 2008.

[9] D. Siringoringo and Y. Fujino, "System identification of suspension bridge from ambient vibration response," Engineering Structures, vol. 30, no. 2, pp. 462-477, 2008.

[10] B. Peeters and G. de Roeck, "Reference based stochastic subspace identification in civil engineering," Inverse Problems in Engineering, vol. 8, no. 1, pp. 47-74, 2000.

[11] J. Brownjohn, F. Magalhaes, E. Caetano, and A. Cunha, "Ambient vibration re-testing and operational modal analysis of the Humber Bridge," Engineering Structures, vol. 32, no. 8, pp. 20032018, 2010.

[12] C. Kim, M. Kawatani, and J. Hao, "Modal parameter identification of short span bridges under a moving vehicle by means of multivariate AR model," Structure and Infrastructure Engineering, vol. 8, no. 5, pp. 459-472, 2012.

[13] P. Andersen, R. Brincker, and P. Kirkegaard, "Theory of covariance equivalent ARMAV models of civil engineering structures," in Proceedings of 14th International Modal Analysis Conference, pp. 518-524, Dearborn, Mich, USA, 1996.

[14] G. S. Urgessa, "Vibration properties of beams using frequencydomain system identification methods," Journal of Vibration and Control, vol. 17, no. 9, pp. 1287-1294, 2011.

[15] R. Brincker, L. Zhang, and P. Andersen, "Modal identification of output-only systems using frequency domain decomposition," Smart Materials and Structures, vol. 10, no. 3, pp. 441-445, 2001.

[16] B. Yan, A. Miyamoto, and E. Brühwiler, "Wavelet transformbased modal parameter identification considering uncertainty," Journal of Sound and Vibration, vol. 291, no. 1-2, pp. 285-301, 2006.
[17] J. Amezquita-Sanchez, A. Garcia-Perez, R. Romero-Troncoso, R. Osornio-Rios, and G. Herrera-Ruiz, "High-resolution spectral-analysis for identifying the natural modes of a trusstype structure by means of vibrations," Journal of Vibration and Control, vol. 19, no. 16, pp. 2347-2356, 2012.

[18] B. Yan and A. Miyamoto, "A comparative study of modal parameter identification based on wavelet and Hilbert-Huang transforms," Computer-Aided Civil and Infrastructure Engineering, vol. 21, no. 1, pp. 9-23, 2006.

[19] S. Chen, J. Liu, and H. Lai, "Wavelet analysis for identification of damping ratios and natural frequencies," Journal of Sound and Vibration, vol. 323, no. 1-2, pp. 130-147, 2009.

[20] J. Tang, D. Chiou, C. Chen, W. Chiang, W. Hsu, and T. Liu, "A case study of damage detection in benchmark buildings using a Hilbert-Huang Transform-based method," Journal of Vibration and Control, vol. 17, no. 4, pp. 623-636, 2010.

[21] T. Liu, W. Chiang, C. Chen, W. Hsu, L. Lu, and T. Chu, "Identification and monitoring of bridge health from ambient vibration data," Journal of Vibration and Control, vol. 17, no. 4, pp. 589-603, 2011.

[22] T. Liu, W. Chiang, C. Chen et al., "Structural system identification for vibration bridges using the Hilbert-Huang transform," Journal of Vibration and Control, vol. 18, no. 13, pp. 1939-1956, 2012.

[23] W. Hsu, D. Chiou, C. Chen, M. Liu, W. Chiang, and P. Huang, "Sensitivity of initial damage detection for steel structures using the Hilbert-Huang transform method," Journal of Vibration and Control, vol. 19, no. 6, pp. 857-878, 2013.

[24] K.-M. Chang, "Arrhythmia ECG noise reduction by ensemble empirical mode decomposition," Sensors, vol. 10, no. 6, pp. 6063-6080, 2010.

[25] Z. Wu and N. Huang, "Ensemble empirical mode decomposition: a noise-assisted data analysis method," Tech. Rep. Series 193, Center for Ocean-Land-Atmosphere Studies, 2005.

[26] X. Jiang and H. Adeli, "Pseudospectra, MUSIC, and dynamic wavelet neural network for damage detection of highrise buildings," International Journal for Numerical Methods in Engineering, vol. 71, no. 5, pp. 606-629, 2007.

[27] R. Osornio-Rios, J. Amezquita-Sanchez, R. Romero-Troncoso, and A. Garcia-Perez, "MUSIC-ANN analysis for locating structural damages in a truss-type structure by means of vibrations," Computer-Aided Civil and Infrastructure Engineering, vol. 27, no. 9, pp. 687-698, 2012.

[28] N. Huang, Z. Shen, S. Long et al., "The empirical mode decomposition and the Hubert spectrum for nonlinear and nonstationary time series analysis," Proceedings of The Royal Society of London A, vol. 454, pp. 903-995, 1998.

[29] Z. Wu and N. E. Huang, "Ensemble empirical mode decomposition: a noise-assisted data analysis method," Advances in Adaptive Data Analysis, vol. 1, no. 1, pp. 1-41, 2009.

[30] A. Garcia-Perez, R. D. J. Romero-Troncoso, E. Cabal-Yepez, and R. A. Osornio-Rios, "The application of high-resolution spectral analysis for identifying multiple combined faults in induction motors," IEEE Transactions on Industrial Electronics, vol. 58, no. 5, pp. 2002-2010, 2011.

[31] K. Young and H. Adeli, "Fundamental period of irregular moment-resisting steel frame structures," The Structural Design of Tall and Special Buildings, 2013. 

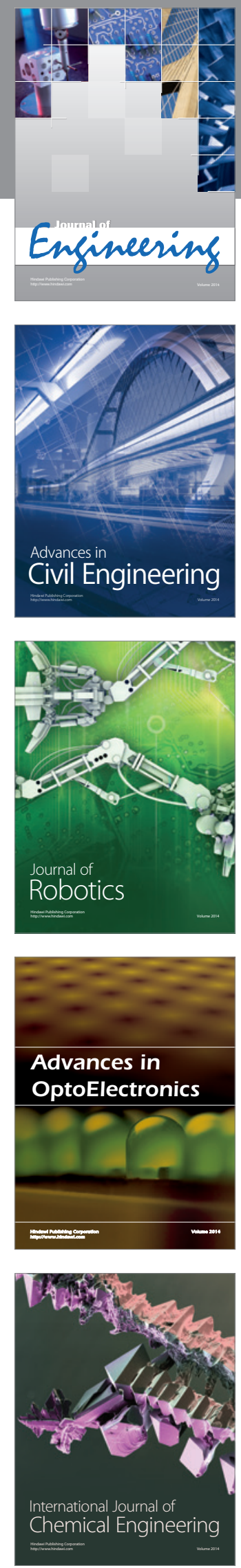

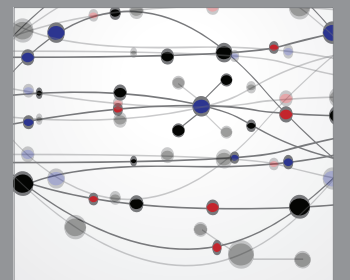

The Scientific World Journal
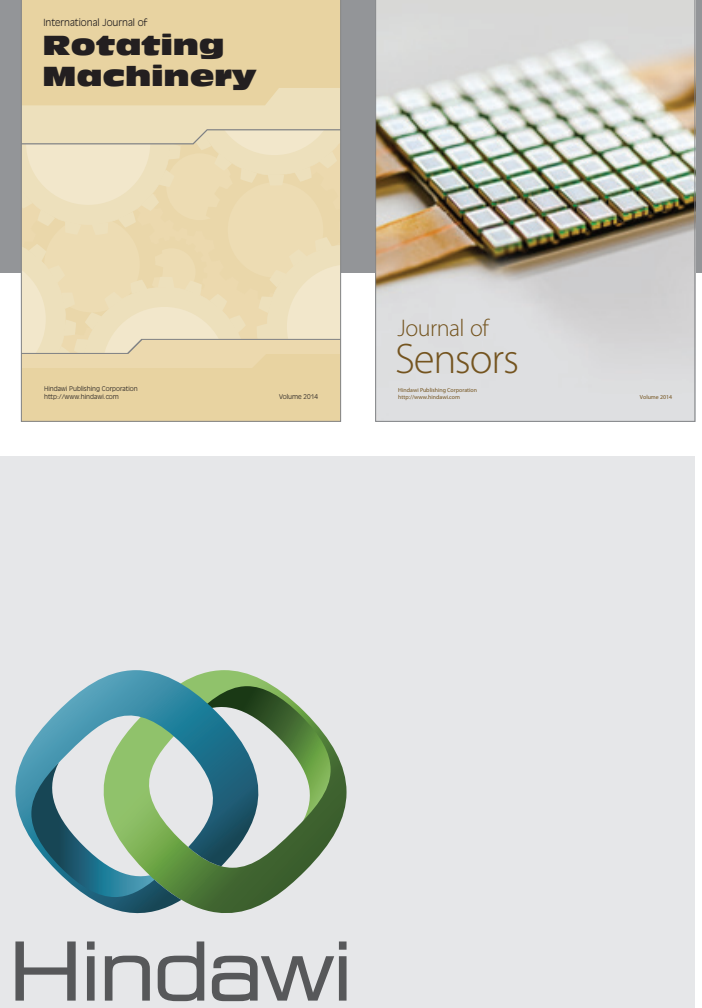

Submit your manuscripts at http://www.hindawi.com
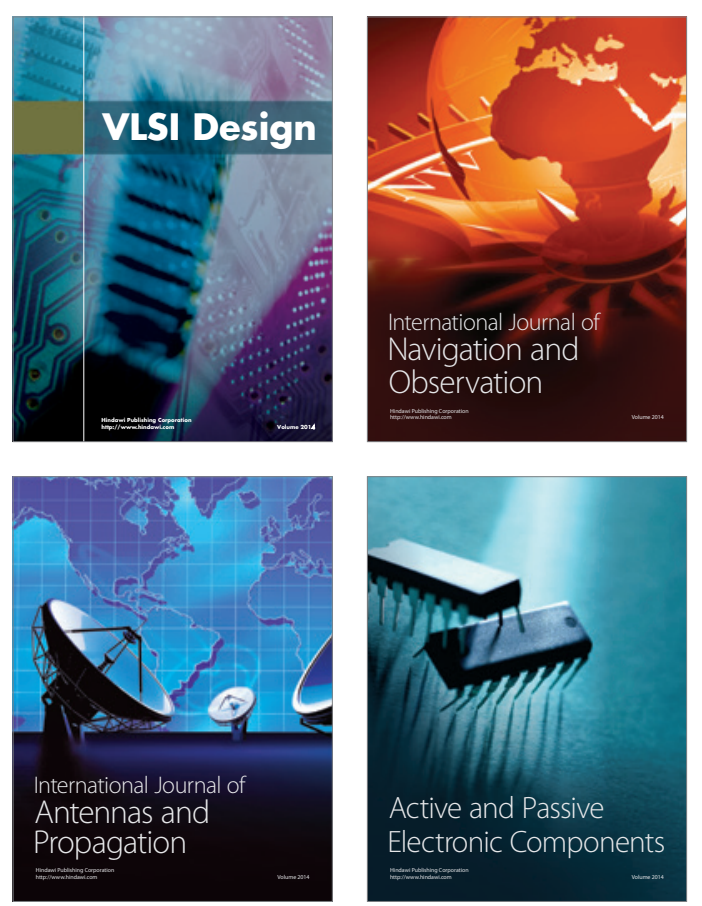
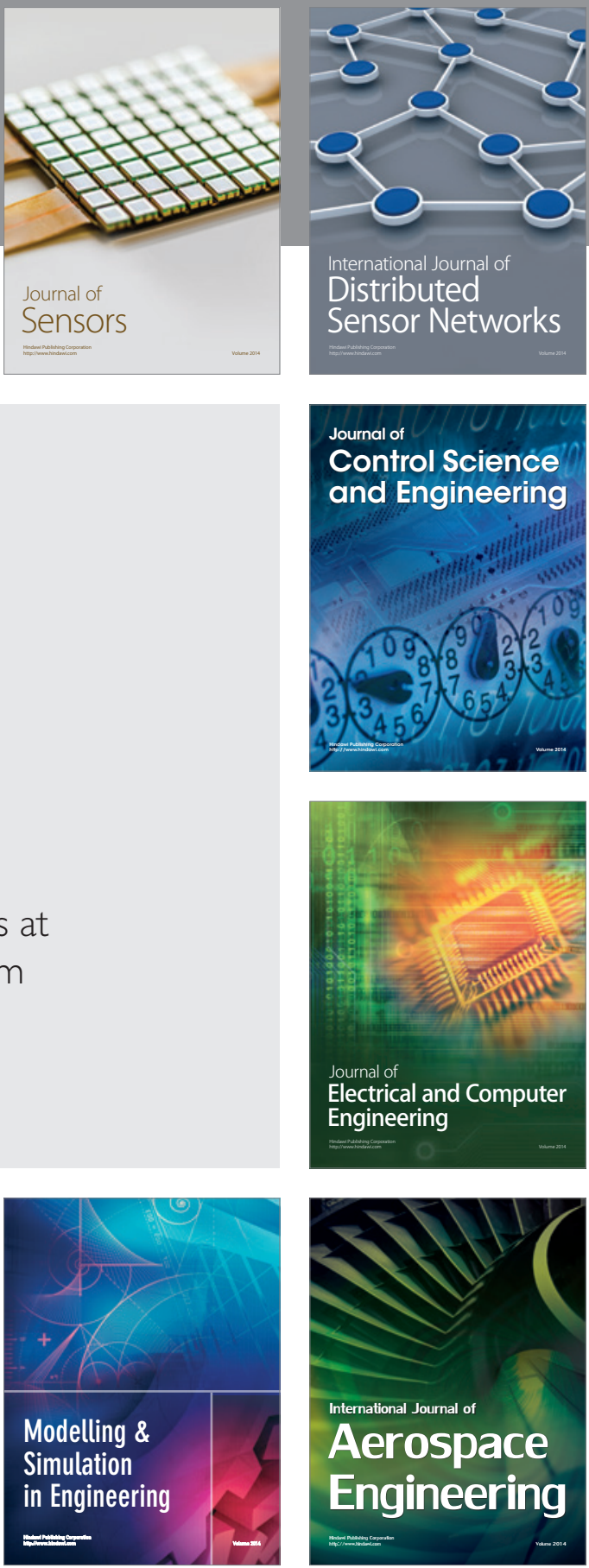

Journal of

Control Science

and Engineering
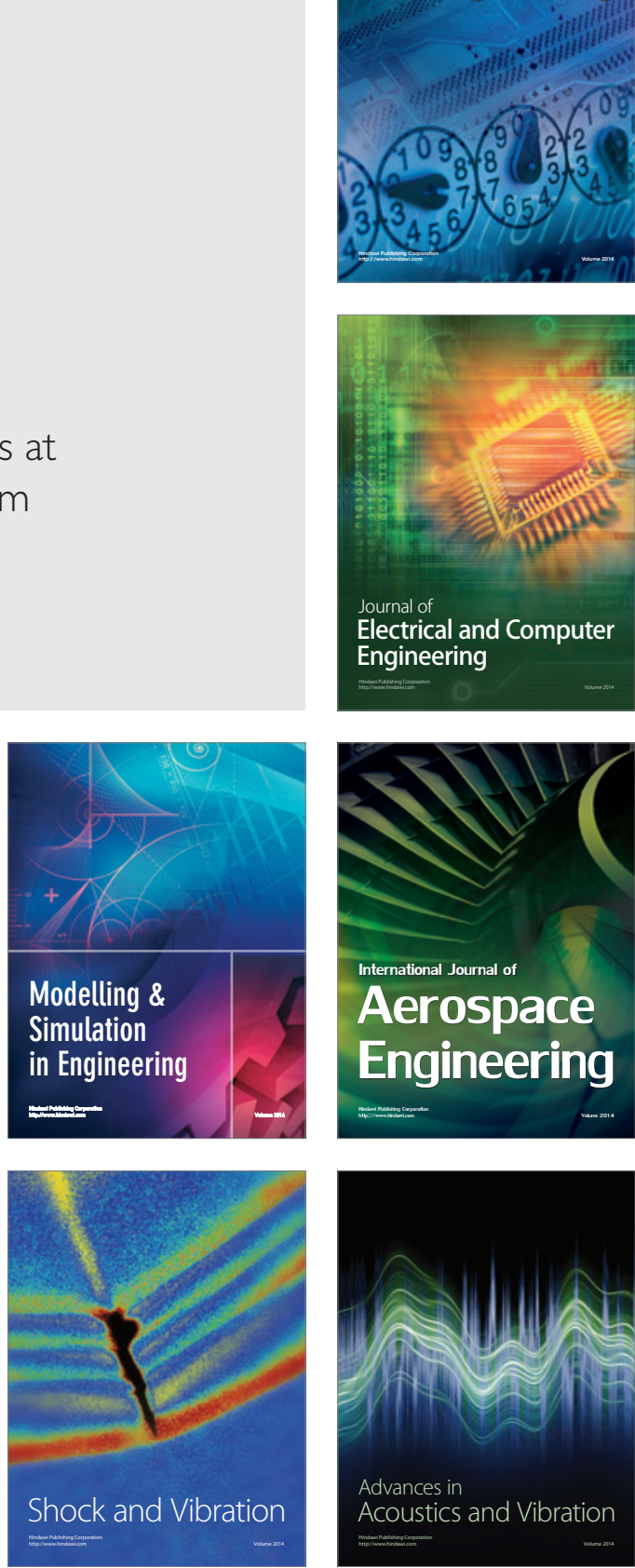